\title{
In situ monitoring of tunnel deformation evolutions from auxiliary tunnel in deep mine
}

\author{
Hui Zhou, Cheng-kun Qu, Da-wei Hu*, Chuan-qing Zhang, Muhammad Usman Azhar, Zheng Shen, Jun Chen \\ State Key Laboratory of Geo-mechanics and Geotechnical Engineering, Institute of Rock and Soil Mechanics, Chinese \\ Academy of Sciences, Wuhan, Hubei 430071, China \\ Corresponding author: Dawei $\mathrm{Hu}$ \\ +86-27-87197913 \\ E-mail address:dwhu@whrsm.ac.cn
}

\begin{abstract}
Deformation caused by excavation is of great effects on tunnel stability and tunnel support design. Monitoring using instruments that are installed in place within the examined tunnel, cannot record a large amount of surrounding rock deformation that usually takes place ahead and close to the tunnel face. For this important reason, the overall deformation process was observed using instruments installed prior to excavation from auxiliary tunnel in a $900 \mathrm{~m}$ deep coal mine tunnel. Two monitoring methods, i.e. multipoint extensometers and wave velocity tomography were used at two monitoring sections with different rock lithology. The results showed that a large amount of deformation at both monitoring sections had already been occurred when the excavation face passed the monitoring sections at a very small distance. The size of the plastic zone at monitoring section 1 is identified as $1.8 \mathrm{~m}(0.36 \mathrm{D}, \mathrm{D}$ denotes the diameter of the examined tunnel) and the velocities within this zone decreased from $4.5 \mathrm{~km} / \mathrm{s}$ to $2 \mathrm{~km} / \mathrm{s}$. The primary deformation was generated in a distance from $-0.8 \mathrm{D}$ (ahead the excavation face) to 2-3 D (behind the excavation face). The induced deformation was also observed after the primary deformation period, and some additional deformation was still occurred in the plastic zone although the size of plastic zone remain constant. The displacements and p-wave velocities at the two monitoring sections showed little differences.
\end{abstract}

Keywords: In situ monitoring, Plastic zone, Multipoint extensometers, P-wave velocity tomography, Induced deformation 


\section{Introduction}

Tunnel excavations in deep rock formations cause re-arrangement of geostatic stresses and consequently generate large deformation in surrounding rocks (Brox and Hagedorn, 1999; Goel et al 1995; Kaiser, 1993; Kontogianni and Stiros, 2002). In the case of using drill and blast excavation method, the mechanical properties of surrounding rocks are considerably changed (Kwon and Cho 2008; Wassermann et al. 2011; Siren et al. 2015; Perras and Diederichs 2015). In areas of surrounding rock mass near the sidewalls of tunnel, significant plastic deformation and obvious variation in term of decrease in wave velocity have been observed (Sato et al. 2000; Martino and Chandler 2004; Yan et al. 2014). In this plastic zone, rock is either irreversibly damaged or disturbed with significant change in physical, mechanical and hydraulic properties (Sato et al. 2000; Bossart et al. 2002; Tsang et al. 2005; Wu et al. 2009; Diederichs et al. 2004; Li and Liu 2013; Lisjak et al. 2014; Siren et al. 2015), and these irreversible variations are directly related to crack initiation, propagation and coalescence (Li et al. 2012; Perras and Diederichs 2015).

Different monitoring methods have been applied to investigate the size and shape of the plastic zone change throughout the excavation process (Kavvadas, 2005). Using micro velocity probe and borehole camera, Martino and Chandler (2004) revealed that the plastic zone was influenced by different factors, e.g. stress adjustment, tunnel direction, shape of excavated section, excavation manner, and other affecting factors. The evolutionary process of cracks in an aquifer was analyzed by Williams and Johnson (2004) using acoustic velocity detection and borehole imaging. Blümling et al. (2007) presented the initial formation stage and long-term processes of plastic zones by microfocus X-ray tomography. Rock bursts in the TBM excavation were monitored via acoustic emission by Cheng et al. (2013). Based on numerical simulations, Yan et al. (2014) proposed that the plastic zones were caused by combined action of blasting load, stress redistribution and transient unloading. Zhu et al. (2014) analyzed the effect of dynamic stress redistribution on plastic zones using a twodimensional mathematical model. Moreover, based on geodetic monitoring data from transportation tunnels and mining galleries, Kontogianni and Stiros (2005) suggested that the deformation is a 3-D effect instead of 2-D effect, there exists an ignored component of deformation along the tunnel axis which is responsible for the propagation of deformation along tens of meters or even more.

In the above studies, the monitoring instruments were mainly installed in place within the examined tunnel as the excavation face advances; and the minimum distance of 
instrument installation from the tunnel face is around 2-4 $\mathrm{m}$ to avoid interference with the construction of the temporary support (sprayed concrete, steel sets, etc) (Kavvadas, 2005). However, a large amount of surrounding rock deformation takes place ahead and close to the tunnel face, from about 1D ahead of the face up to about 1.5D behind the face (Hoek, 2001; Kontogianni and Stiros, 2002; Kavvadas, 2005). Therefore, an appreciable portion of the actual deformation occurred prior to instrument installation, cannot be recorded (Kavvadas, 2005). Thus, monitoring using instruments installed from neighboring tunnel prior to excavation of examined tunnel, is highly important for the overall deformation process of plastic zone. Moreover, different from tunnel excavation in hydropower or transportation engineering in the above studies, tunnels excavated in coal mines usually encounters weak clastic rocks (sandstones, claystones, mudstones, and conglomerates) that are characterized by low mechanical strength, high deformability and significant structural anisotropy, which can also influence the development of plastic zone. For this purpose, two monitoring instruments, multi-point extensometer and wave velocity tomography, were installed from the auxiliary tunnel prior to excavation of examined tunnel in a $900 \mathrm{~m}$ deep tunnel of coal mine. The overall process of plastic zone was observed at two monitoring sections with different rock lithology.

\section{Site Descriptions and monitoring scheme}

\subsection{Site Descriptions}

The monitoring studies were performed in Jining coal mine, located at eastern part of China. The examined tunnel, $5 \mathrm{~m}$ in diameter, was excavated at a depth of $900 \mathrm{~m}$ using drill and blast excavation method. The examined area is about $160 \mathrm{~m}$ long from line $\mathrm{X}$ to line $\mathrm{Y}$ (see Fig.1). The support system included shot concrete, steel sets, and occasionally rock bolts. In order to install monitoring instruments prior to the excavation of examined tunnel, an auxiliary tunnel, whose axis is parallel to the examined tunnel axis, was excavated using drill and blast technique in the neighboring rockmass prior to the excavation of examined tunnel. The layout of the two tunnels is presented the in Fig. 1. The distance between the two tunnels is about $40 \mathrm{~m}$, which is $8 \mathrm{D}$ and sufficiently large to avoid the interactions between the two tunnels.

The principal strata is mainly sandstone of Shanxi Group. The sandstone formation displays an obvious horizontal bedding (see Fig. 2). The elastic modulus and Poisson's ratio of the rock mass near the examined tunnel are approximately $18-35 \mathrm{GPa}$ and $0.24-0.28$, respectively. Compressive strength of intact rock determined by laboratory tests is in between 
80 and $120 \mathrm{MPa}$, and the rock quality designation (RQD) value (Deere, 1988) is about 45\%$61 \%$.

The in situ stress of rock mass is presented in the Table 1. The three principal stresses are 19.5, 9.5 and $8.4 \mathrm{MPa}$, respectively. The maximum principal stress $\sigma_{1}$ and intermediate principal stress $\sigma_{2}$ are close to the horizontal direction, and the minimum principal stress $\sigma_{3}$ is at $38^{\circ}$ deviated from the vertical direction.

Two monitoring sections (see Fig. 1) were selected in order to analyze the effect of lithological difference on the plastic zone. Monitoring sections 1 and 2 were respectively located in medium-grain sandstone and siltstone, 40 and $100 \mathrm{~m}$ ahead of the initial tunnel excavation face along the axis of the examined tunnel. Cylindrical rock samples of the medium grain sandstone and siltstone with dimensions $50 \times 100 \mathrm{~mm}$ were acquired from the site for laboratory testing. The uniaxial compression test was conducted on an MTS testing machine according to ISRM suggest method (ISRM, 2007) and the typical stress-strain curves obtained are presented in Fig. 3. The strength and elastic modulus of the medium grain sandstone are $120 \mathrm{MPa}$ and $17.1 \mathrm{GPa}$ respectively, which are slightly greater than that (107 $\mathrm{MPa}$ and $11.9 \mathrm{MPa}$ ) of the siltstone. This difference in deformation characteristics will be considered in evaluating the following monitoring results.

\subsection{Monitoring scheme}

In this work two different monitoring instruments, i.e., multi-point extensometers and wave velocity tomography, were installed respectively from the auxiliary tunnel prior to the excavation of the examined tunnel. The multi-point extensometers were installed in the boreholes at different pre-selected depths away from the examined tunnel sidewall, and the boreholes were consequently backfilled with cement-bentonite grout. Therefore, the multipoint extensometers measured the relative displacement along the boreholes at discrete intervals from the borehole bottom to the exanimated tunnel sidewall. Generally, the multipoint extensometers have a limit measuring range (i.e. 100-300 mm), which is the major shortcoming comparing with geodetic monitoring (Kavvadas, 2005). In this study, the measuring accuracy and range of multi-point extensometer are $0.01 \mathrm{~mm}$ and $100 \mathrm{~mm}$, respectively, and this monitoring range is sufficiently large comparing with the maximum displacement in the plastic zone. Five discrete measuring points were selected in each borehole. At monitoring section 1, the five measuring points were located at $0.5,2,5,8,10 \mathrm{~m}$ away from the exanimated tunnel sidewall, respectively. However, the monitoring results 
indicated that the displacements of measuring points at depth beyond $8 \mathrm{~m}$ were close to zero. Therefore, a measuring point at $1 \mathrm{~m}$ was replaced instead of $10 \mathrm{~m}$ in monitoring section 2 .

Considering the discrete nature of the measuring points using the multi-point extensometer, it is thus difficult to identify the accurate size and shape of the plastic zone. Therefore, the wave velocity (P-wave) tomography method was further applied. The instrument is equipped with one transmitter and two receivers with acoustic pulses of $30 \mathrm{kHz}$ and moves along the detecting borehole with an interval of $0.2 \mathrm{~m}$ from borehole bottom to tunnel sidewall (Yan et al. 2014). The transmitter produces a P-wave pulse, and the two receivers, which are located at distance of 30 and $50 \mathrm{~mm}$ with respect to the transmitter, that receive the produced pulse via the borehole fluid and rockmass (see Fig. 4). The P-wave velocity of the rockmass along the whole length of the borehole is consequently obtained. Due to that the velocity of ultrasonic pulses travelling in a solid material depends on the density and as well elastic properties of that material. The quality of some materials is related to their elastic stiffness so that measurement of ultrasonic pulse velocity in such materials can often be used to indicate their quality. The wave velocity tomography has been applied to predict rock mass deformation as well as the extent of plastic zones developed around underground openings (Barton, 2007). In this study, the results obtained from the wave velocity tomography were compared with the results of multi-point extensometer in order to further investigate the plastic zone.

As mentioned above, the monitoring investigations were performed from the auxiliary tunnel prior to excavation of examined tunnel. The boreholes for installation of multi-point extensometer and wave velocity tomography were drilled from the auxiliary tunnel to the sidewall of the examined tunnel (Fig. 5). For the boreholes of wave velocity tomography, it is required to fill the borehole with water, so a distance of $0.8 \mathrm{~m}$ was kept between the borehole bottoms and the sidewall of the examined tunnel. The diameter of the boreholes using for the multi-point extensometer and wave velocity tomography is $56 \mathrm{~mm}$, and the borehole spacing is about $1 \mathrm{~m}$ in order to avoid their interactions. The arrangements of the boreholes are presented in Fig. 5.

\section{Monitoring results and analysis}

This section will present the monitoring results in two different monitoring sections using the multi-point extensometer and wave velocity tomography from the auxiliary tunnel during excavation of examined tunnel.

\subsection{Displacement monitoring results}


Fig. 6 shows the displacement evolution curves obtained from the multi-point extensometers installed at different depths in boreholes at the two monitoring sections, i.e. monitoring section 1 with medium-grain sandstone and monitoring section 2 with siltstone. The results indicate significant displacement occurs in the surrounding rocks near the sidewalls during the excavation. The measured displacement decreased with increasing depth from the sidewall and approaches to zero at depths $5 \mathrm{~m}$ and $8 \mathrm{~m}$ respectively at monitoring section 1 and 2. As mentioned above, the size of the plastic zone is difficult to identify from Fig. 6 due to the discrete nature of the measuring points using the multi-point extensometer. Therefore, the wave velocity monitoring will be further applied to calibrate the size of the plastic zone. At monitoring sections 1 and 2, the maximum displacements observed were approximately $19 \mathrm{~mm}$ and $22 \mathrm{~mm}$ respectively by the measuring point at $0.5 \mathrm{~m}$ depth. The difference in displacement recorded by measuring points located at $0.5 \mathrm{~m}$ and $2 \mathrm{~m}$ depths was significant while measuring points at $5 \mathrm{~m}, 8 \mathrm{~m}$ and $10 \mathrm{~m}$ depth observed small displacement in monitoring section 1 . Therefore, to better observe the displacement of the surrounding rocks near the sidewalls, a measuring point at $1 \mathrm{~m}$ depth was added instead of $10 \mathrm{~m}$ depth at monitoring section 2 . The results from monitoring section 2 show the similar displacement characteristics as that from monitoring section 1. It is noted that the displacement of measuring point of $2 \mathrm{~m}$ depth at monitoring section 2 shows a fluctuation when the excavation face passed the monitoring section by $32 \mathrm{~m}$. This fluctuation can be attributed to the adhesion loose of the measuring point given the other four points do not show any influence at the same time.

With the advancing of the excavation face, the displacements at both measuring sections undergo both increase and stabilization process. The measuring points near the sidewall start to produce a small disturbance, e.g. the onset of displacement, when the tunnel excavation face approaches to the monitoring sections (i.e. the monitoring sections are about $4 \mathrm{~m}$ ahead of the excavation face at both monitoring sections). The displacements of the measuring points near the sidewall (i.e. $0.5 \mathrm{~m}$ depth from the sidewall) gradually increase in a large rate when the excavation face arrived at the monitoring section and passed through the monitoring section by $5-10 \mathrm{~m}$, consequently increase in small rate after the excavation face passing through the monitoring section by 15 and $12 \mathrm{~m}$ respectively at monitoring sections 1 and 2 . After this period, the displacements of measuring points beyond a depth of $2 \mathrm{~m}$ at both monitoring sections are nearly stable, while the displacements of measuring points with a depth of $0.5 \mathrm{~m}$ at both monitoring sections still continue to increase in a slight rate. 
As mentioned above, most of the previous monitoring instruments were mainly installed within the tunnel behind the tunnel face as the tunnel advances (Sato et al. 2000; Martino and Chandler 2004; Yan et al. 2014). Therefore, the monitoring methods within the examined tunnel are unable to record the deformation generated before the installation of the monitoring instruments. A comparison between displacements at different depths from sidewalls which were generated during overall excavation process, and $5 \mathrm{~m}$ after the excavation face passed the monitoring sections is shown in Fig. 7. The displacements at the measuring points near the sidewall (e.g. at 0.5, 1, 2 and $5 \mathrm{~m}$ depth) of both monitoring sections generated in the latter case are significantly smaller than those in the former case. The differences of the displacements of measuring point of $0.5 \mathrm{~m}$ depth between the two cases account for $37 \%$ and $45 \%$ of the total displacements at monitoring sections 1 and 2 , respectively. This confirmed the monitoring results observed by Hoek (2001), Kontogianni and Stiros (2002), and Kavvadas (2005).

Moreover, a first assessment of the boundary of plastic zone size is performed based on the monitoring results from the multi-point extensometers. For clarification, Fig. 6 was replotted to compare the displacement gradients between adjacent measuring points, as shown in Fig. 8. At monitoring section 1, with the increase in depth, the displacements of measuring points significantly decreased in the zone within the depth of $5 \mathrm{~m}$ and became nearly stable beyond this zone. This significant variation in displacement, which is mainly caused by the irreversible variations related to crack initiation, propagation and coalescence (Li et al. 2012; Perras and Diederichs 2015), is considered as an indicator of plastic zone. Therefore, the boundary of the plastic zone at monitoring section 1 is firstly assessed within 5 $\mathrm{m}$. Similar tendency can be seen in section 2, the displacements of measuring points significantly decreased in the zone within the depth of $2 \mathrm{~m}$, while decreased much lesser beyond this zone. The boundary of the plastic zone at monitoring section 2 is thus within $2 \mathrm{~m}$. Considering the discrete nature of multi-point extensometers results, it is just preliminary assessment and needs further validation.

\subsection{Wave velocity monitoring results}

The monitoring results of the two monitoring sections using the p-wave velocity tomography are presented in Fig. 9. As a distance of $0.8 \mathrm{~m}$ was kept between borehole bottom and the sidewall of the examined tunnel for the filling of water in borehole, the p-wave velocities $\left(\mathrm{V}_{\mathrm{p}}\right)$ in this region cannot be obtained (see Figs. 3 and 9). At monitoring section 1 (see Fig. 9(a)), 
significant variations in wave velocity were observed in the surrounding rock mass near sidewall during the excavation process. During the tunnel excavation face reached at the monitoring section (i.e. the monitoring sections are about $3 \mathrm{~m}$ and $0 \mathrm{~m}$ ahead of the tunnel excavation face), the p-wave velocities remained unchanged at various depths from the tunnel sidewall, and this velocity was about 4.5-5 km/s. According to the relationship $\left(\mathrm{V}_{\mathrm{p}} \approx 3.5+\log _{10} \mathrm{Q}\right)$ between $\mathrm{V}_{\mathrm{p}}$ and Q-value rock quality (Barton, 2007), the Q-value of the rockmass under original state is in the range of 10-32, which is consistent with the RQDvalue as mentioned in Section 2. Consequently, the velocity within a zone of $1.8 \mathrm{~m}$ depth significantly decreased when the tunnel excavation face passed the monitoring section (i.e. the monitoring section is about $6 \mathrm{~m}$ behind the tunnel excavation face), and finally approached to a constant value (about $2 \mathrm{~km} / \mathrm{s}$ ) (i.e. the monitoring section is $26 \mathrm{~m}$ behind the tunnel excavation face).

At monitoring section 2 (see Fig. 9(b)), there was no significant variation in wave velocity observed when the monitoring section was $10 \mathrm{~m}$ and $2 \mathrm{~m}$ ahead of the tunnel excavation face. Therefore, it can be concluded that the rock mass $2 \mathrm{~m}$ ahead of tunnel excavation face remained uninfluenced. Unfortunately, the borehole bottom got connected with the sidewall of the examined tunnel due to blasting effect, and it failed to fill the borehole with water. Therefore, the wave velocity monitoring cannot be performed after the excavation face reached at the monitoring section.

As mentioned above, the velocity of ultrasonic pulses travelling in a solid material depends on the density and elastic properties of that material. The reduction in p-wave velocities is directly related to the blasting damage and stress redistribution caused by excavation (Maxwell et al. 1998; Barton, 2007). Therefore, the zone where p-wave velocities significantly fall down is considered as the plastic zone. For monitoring section 1, the velocities within a zone of $1.8 \mathrm{~m}$ depth decreased from $4.5 \mathrm{~km} / \mathrm{s}$ to $2 \mathrm{~km} / \mathrm{s}$ during the excavation process, the size of the plastic zone at monitoring section 1 is identified as $1.8 \mathrm{~m}$ $(0.36 \mathrm{D})$. The accurate size of plastic zone at monitoring section 2 is unknown due to the lack of monitoring results.

\subsection{Discussions}

First, the deformation evolution at the two monitoring sections has been studied during the advancing process of the excavation face. Previous in-situ monitoring results (Kontogianni and Stiros 2005; Stiros and Kontogianni 2009) indicated that the deformation of 
surrounding rocks caused by excavations may be combined with two parts: primary deformation and induced deformation. The former one is confined to a certain volume of rocks around the excavation face and can be determined based on Saint Venant's rule. This volume is usually located at a distance of 2-3 D backward the excavations face along the tunnel axis (Kontogianni and Stiros 2005; Stiros and Kontogianni 2009; Sato et al. 2000). In this study, the displacements nearly reach stability after the excavation face passing through the monitoring section by $3 \mathrm{D}$ and $2.4 \mathrm{D}$ at monitoring section 1 and 2 , respectively, which coincide with the previous monitoring results. However, the onset of displacement was already observed when the excavations face didn't yet arrived at the monitoring sections (i.e. the monitoring section are about $0.8 \mathrm{D}$ ahead the excavation face at both monitoring sections). Therefore, the present monitoring results indicate that the primary deformation is generated in a volume from -0.8 D (ahead the excavation face) to 2-3 D (behind the excavation face). The latter one is usually ignored and caused propagation deformation at areas remote from the excavation face (Kontogianni and Stiros 2005). In this study, the displacements of measuring points with a depth of $0.5 \mathrm{~m}$ at both monitoring sections still continue to increase in a slight rate after the excavation face passed through the monitoring section by 2-3 $\mathrm{D}$. Therefore, the present study confirms the existence of this induced deformation.

Second, after the excavation face passing the monitoring section by $6 \mathrm{~m}(1.2 \mathrm{D})$ at monitoring section 1 , the size of the plastic zone is nearly stable (i.e. $1.8 \mathrm{~m}, 0.36 \mathrm{D}$ ), while the displacement in this zone still undergo variations with the advancement of the excavation face (see Figs. 8(a) and 9(a)). In other words, there are still some additional deformation occurred in the plastic zone although the size of plastic zone is constant. However, Li et al. (2012) indicated that the p-wave velocity remained unchanged as soon as the plastic zone size becomes stable. This difference may be attributed to the support method used for the coal mine, which cannot offer adequate support to resist excavation disturbance.

\section{Conclusions}

Two monitoring instruments, multi-point extensometer and wave velocity tomography, were installed from the auxiliary tunnel prior to excavation of examined tunnel in a $900 \mathrm{~m}$ deep tunnel of coal mine. The overall process of plastic zone was observed at two monitoring sections with different rock lithology. A large part of deformation, i.e. respectively $37 \%$ and $45 \%$ of the total displacements of measuring point of $0.5 \mathrm{~m}$ depth at monitoring sections 1 and 2 , had already been occurred when the excavation face passed the monitoring sections by 
$1 \mathrm{D}$. The primary deformation was generated in a distance from $-0.8 \mathrm{D}$ (ahead the excavation face) to 2-3 D (behind the excavation face) and the induced deformation was also observed after the excavation face passed the monitoring sections at a remote distance. The size of the plastic zone at monitoring section 1 is identified as $0.36 \mathrm{D}$ and the velocities within this zone decreased from $4.5 \mathrm{~km} / \mathrm{s}$ to $2 \mathrm{~km} / \mathrm{s}$ respectively. There are still some additional deformation occurred in the plastic zone although the size of plastic zone is constant. The displacements and p-wave velocities at the two monitoring sections show little variations in experimental values.

\section{Acknowledgements}

Financial support from the National Program on Key Basic Research Projects of China (No. 2014CB046902), the National Science Foundation of China (Nos. 51279201, 51479193, 51209085 and 41172288) and the Hundred Talent Program from the Chinese Academy of Sciences are gratefully acknowledged.

\section{References}

Barton, N., 2007. Rock quality, seismic velocity, attenuation and anisotropy. International Journal of Rock Mechanics \& Mining Sciences 44.8:1172.

Bayoumi, A., 2011. On the evaluation of settlement measurements using borehole extensometers. Geotech Geol Eng 29(1),75-90.

Bossart, P., Meier, P.M., Moeri, A., Trick, T., Mayor, J.C., 2002. Geological and hydraulic characterisation of the excavation disturbed zone in the Opalinus Clay of the Mont Terri Rock Laboratory. Eng Geol 66(1-2), 19-38.

Blümling, P., Bernier, F., Lebon, P., Martin, C.D., 2007. The excavation damaged zone in clay formation time-dependent behaviour and influence on performance assessment. Phys Chem Earth, Parts A/B/C ; 32: 588-99.

Brox, D., Hagedorn, H., 1999. Extreme Deformation and Damage during the construction of large tunnels, Tunn Undergr Sp Tech, 14(1), 23-28.

Cheng, W.W., Wang, W.Y., Huang, S.Q., Ma P., 2013. Acoustic emission monitoring of rockbursts during TBM-excavated headrace tunneling at Jinping II hydropower station. Journal of Rock Mechanics and Geotechnical Engineering 5(6), 486-494.

Deere, D. (1988). The rock quality designation (RQD) index in practice. In Rock classification systems for engineering purposes. ASTM International. 
Diederichs, M.S., Kaiser, P.K., Eberhardt, E., 2004. Damage initiation and propagation in hard rock during tunnelling and the influence of near-face stress rotation. Int J Rock Mech Min, 41(5), 785-812.

Goel, R.K., Jethwa, J.L., Paithankar, A.G., 1995. Tunnelling through the young Himalayas A case history of the Maneri - Uttarkashi power tunnel, Eng Geol, 39, 31-44

Hoek E., 2001. Big tunnels in bad rock. J Geotech Geoenviron 127 (9), 726- 740.

ISRM, 2007. The complete ISRM suggested methods for rock characterization, testing and monitoring: 1974-2006. International Soc. for Rock Mechanics, Commission on Testing Methods.

Kaiser, P.K, 1993. Deformation Monitoring for Stability Assessment of Underground Openings, Compressive Rock Engineering, Bergamon, 4, 607-630

Kavvadas, M.J., 2005. Monitoring ground deformation in tunnelling: current practice in transportation tunnels. Eng Geol, 79(1), 93-113.

Kwon, S., Cho, W.J., 2008. The influence of an excavation damaged zone on the thermalmechanical and hydro-mechanical behaviours of an underground excavation. Eng Geol 101(3-4), 110-123.

Kontogianni, V.A., Stiros, S.C., 2002. Predictions and observations of convergence in shallow tunnels: case histories in Greece. Eng Geol, 63(3), 333-345.

Kontogianni, V.A., Stiros S.C., 2005. Induced deformation during tunnel excavation: evidence from geodetic monitoring. Eng Geol 79(1), 115-126.

Li, S.J., Feng, X.T., Li, Z.H., Chen, B.R., Zhang, C.Q., Zhou, H., 2012. In situ monitoring of rockburst nucleation and evolution in the deeply buried tunnels of Jinping II hydropower station. Eng Geol, 137, 85-96.

Li L.C., Liu H.H., 2013. A numerical study of the mechanical response to excavation and ventilation around tunnels in clay rocks. Int J Rock Mech Min 59, 22-32.

Lisjak A., Grasselli G., Vietor T., 2014. Continuum-discontinuum analysis of failure mechanisms around unsupported circular excavations in anisotropic clay shales. Int $\mathbf{J}$ Rock Mech Min 65, 96-115.

Maxwell S.C., Young R.P., Read R.S., 1998. A micro-velocity tool to assess the excavation damaged zone. Int J Rock Mech Min 35(2), 235-247.

Martino J.B., Chandler N.A., 2004. Excavation-induced damage studies at the Underground Research Laboratory. Int J Rock Mech Min 41(8), 1413-1426. 
Perras M.A., Diederichs M.S., 2015. Predicting excavation damage zone depths in brittle rocks. Journal of Rock Mechanics and Geotechnical Engineering, doi:10.1016/j.jrmge.11.004.

Sato T., Kikuchi T., Sugihara K., 2000. In-situ experiments on an excavation disturbed zone induced by mechanical excavation in Neogene sedimentary rock at Tono mine, central Japan. Eng Geol 56(1-2), 97-108.

Siren T., Kantia P., Rinne M., 2015. Considerations and observations of stress-induced and construction-induced excavation damage zone in crystalline rock. Int J Rock Mech Min $73,165-174$.

Stiros S.C., Kontogianni V.A., 2009. Coulomb stress changes: from earthquakes to underground excavation failures. Int J Rock Mech Min, 46(1), 182-187.

Tsang C.F., Bernier F., Davies C., 2005. Geohydromechanical processes in the Excavation Damaged Zone in crystalline rock, rock salt, and indurated and plastic clays-in the context of radioactive waste disposal. Int J Rock Mech Min 42(1), 109-125.

Williams J.H., Johnson C.D., 2004. Acoustic and optical borehole-wall imaging for fracturedrock aquifer studies. J Appl Geophys 55(1-2), 151-159.

Wu F.Q., Liu J.Y., Liu T., Zhuang H.Z., Yan C.G., 2009. A method for assessment of excavation damaged zone (EDZ) of a rock mass and its application to a dam foundation case. Eng Geol 104(3-4), 254-262.

Wassermann J., Sabroux J.C., Pontreau S., et al., 2011. Characterization and monitoring of the excavation damaged zone in fractured gneisses of the Roselend tunnel, French Alps. Tectonophysics 503(1-2), 155-164.

Yan P., Lu W.B., Chen M., et al., 2014. Contributions of In-Situ Stress Transient Redistribution to Blasting Excavation Damage Zone of Deep Tunnels. Rock Mech Rock Eng Doi:10.1007/s00603-014-0571-3

Zhu W.C., Wei J., Zhao J., Niu L.L., 2014. 2D numerical simulation on excavation damaged zone induced by dynamic stress redistribution. Tunn Undergr Sp Tech 43, 315-326. 
Table1 In situ stresses in the monitoring zone.

\begin{tabular}{|c|c|c|c|}
\hline $\begin{array}{c}\text { Principle } \\
\text { stress }\end{array}$ & $\begin{array}{c}\text { Value } \\
(\mathrm{MPa})\end{array}$ & $\begin{array}{c}\text { Azimuth angle } \\
\left({ }^{\circ}\right)\end{array}$ & $\begin{array}{c}\text { Dip angle } \\
\left({ }^{\circ}\right)\end{array}$ \\
\hline$\sigma_{1}$ & 19.5 & 116 & 11.6 \\
\hline$\sigma_{2}$ & 9.5 & 201 & 18.2 \\
\hline$\sigma_{3}$ & 8.4 & 156 & 52 \\
\hline
\end{tabular}



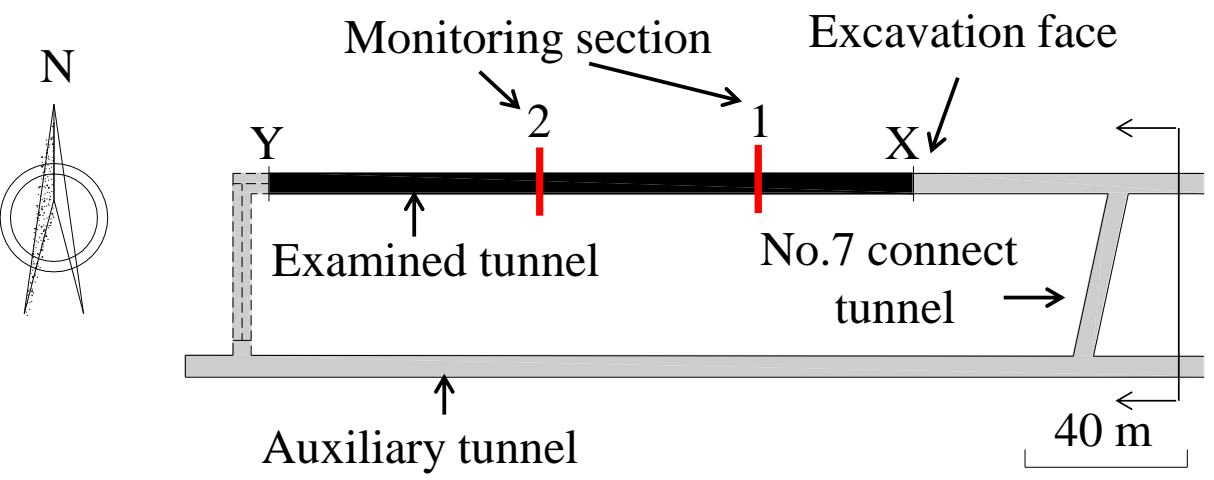

(a) Plan view of two tunnels and monitoring sections

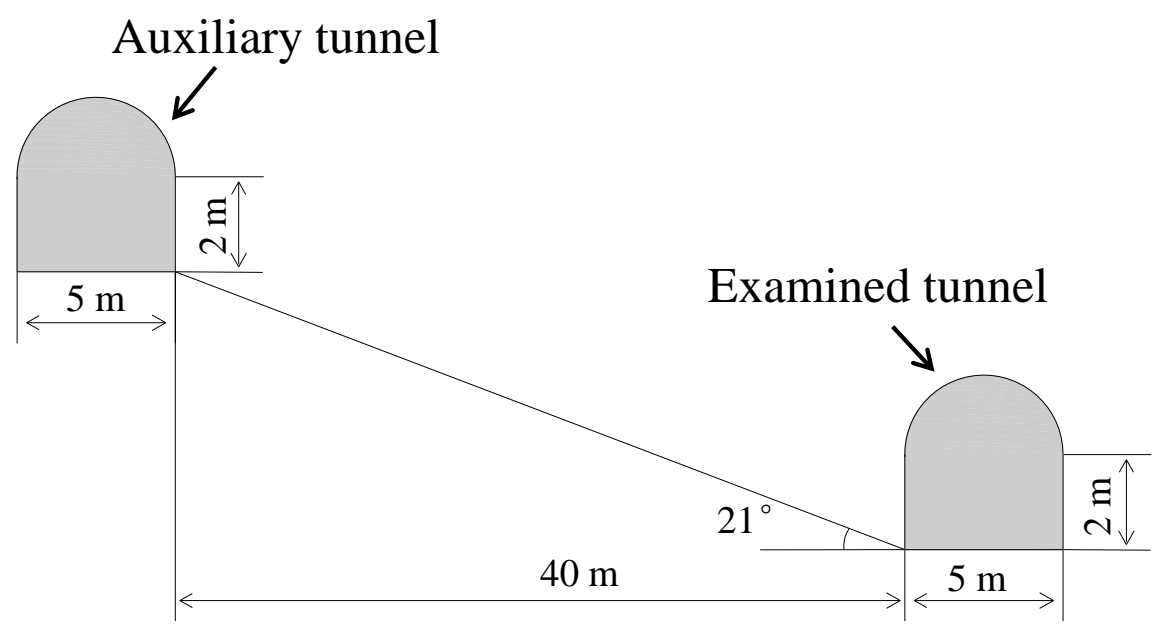

(b) Profile vertical cross section of two tunnels

Fig. 1 Layout of the examined tunnel and auxiliary tunnel 


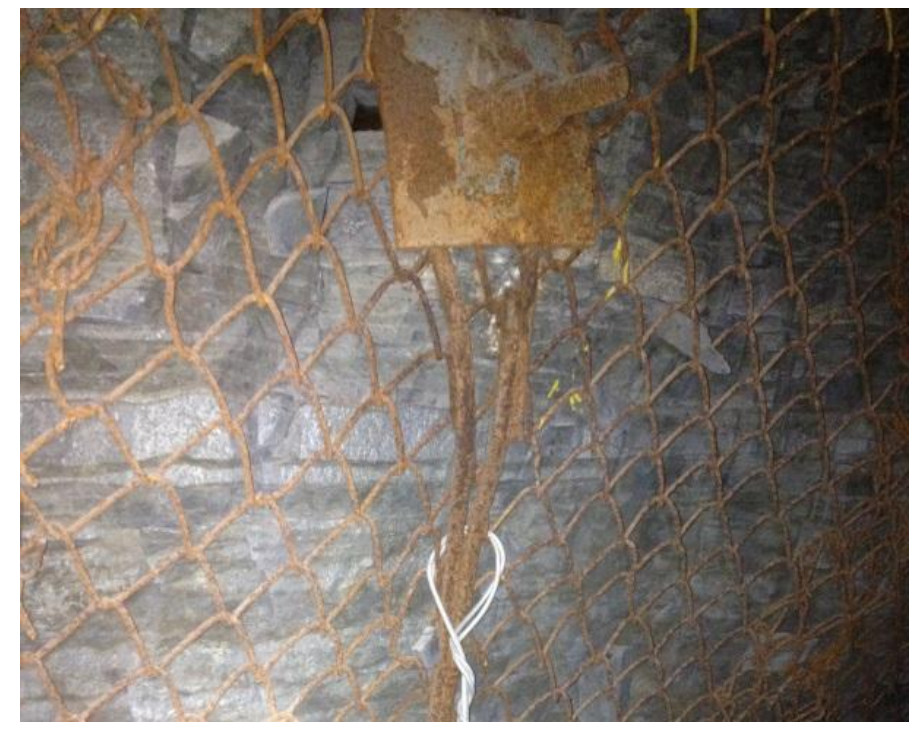

Fig. 2 Horizontal bedding in the monitoring tunnel 


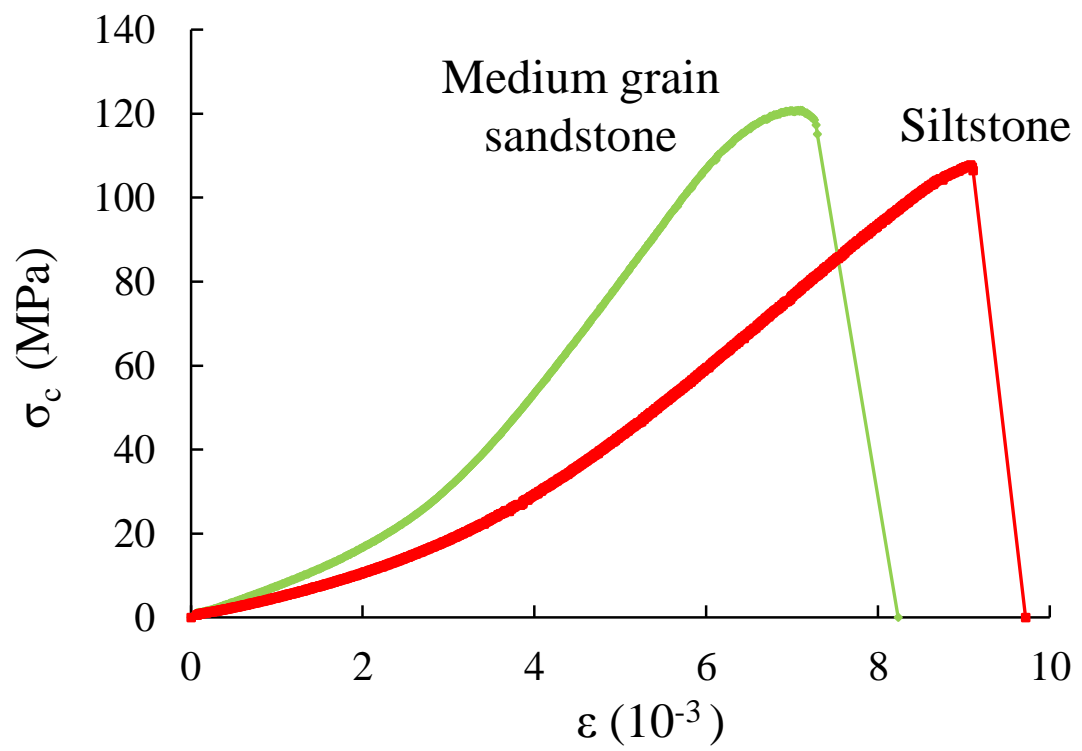

Fig. 3 Stress-strain curves of uniaxial compression of medium-grain sandstone and siltstone 


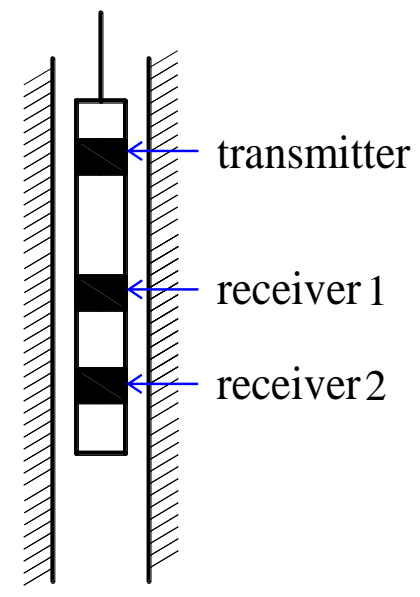

Fig. 4 Schematic diagram of instrumentation for p-wave velocity tomography 


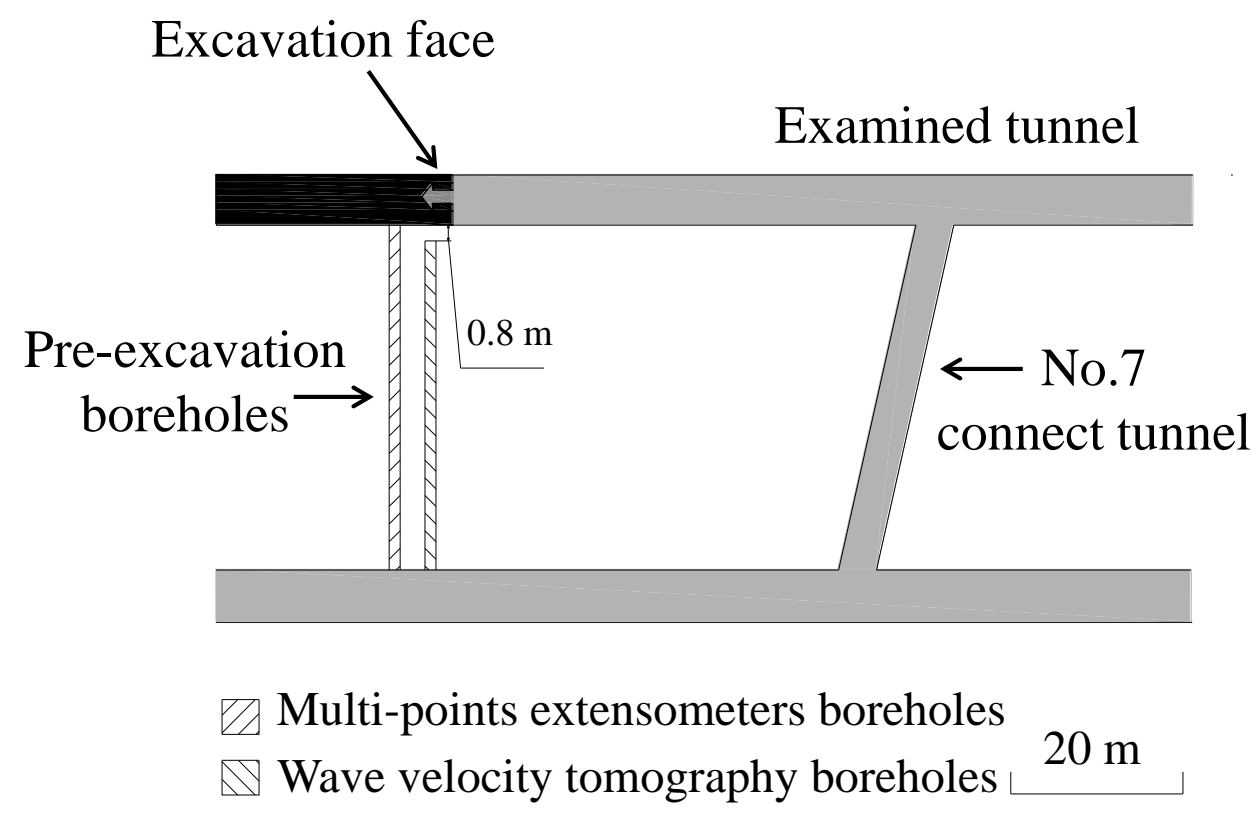

Fig. 5 Schematic diagram of the borehole arrangements 


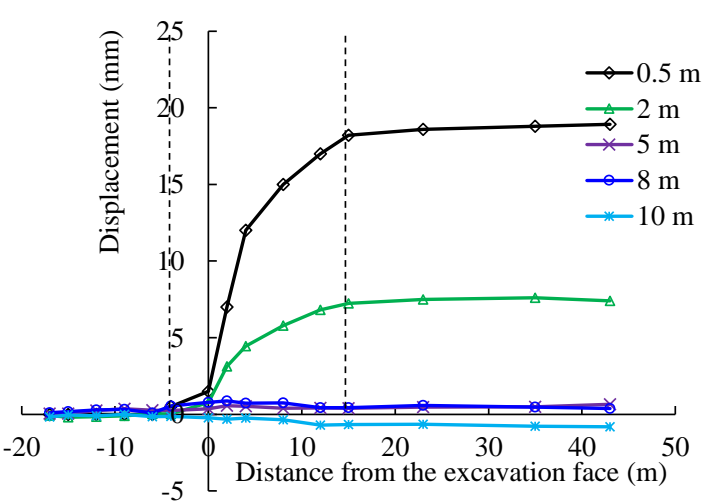

(a) Monitoring section 1

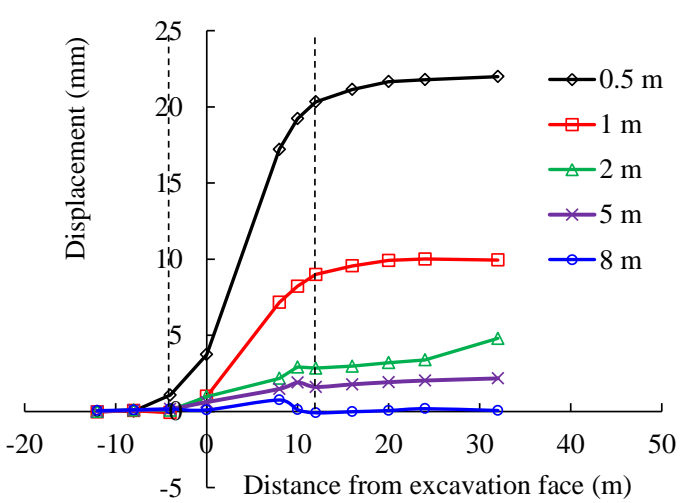

(b) Monitoring section 2

Fig. 6 Displacement evolutions of different measuring points. (E.g. " 0.5 $\mathrm{m}$ " in legend means the measuring point is at $0.5 \mathrm{~m}$ depth away from the sidewall of examined tunnel. With the advancing of the excavation face, the displacements at both measuring sections undergo increase and stabilization process) 


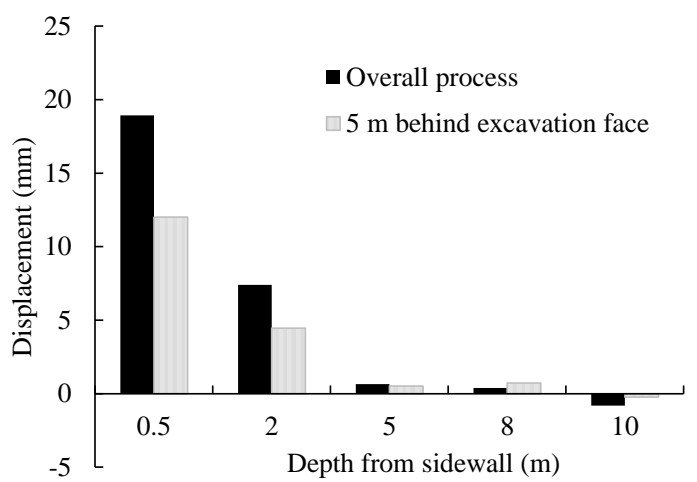

(a) Monitoring section 1

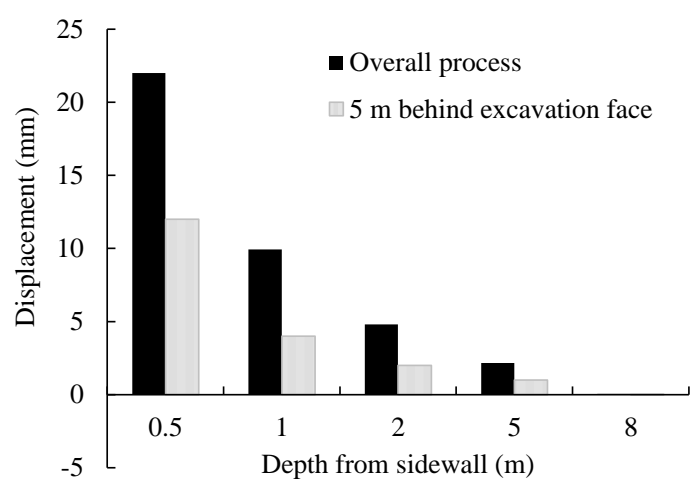

(b) Monitoring section 2

Fig. 7 Comparisons of displacements generated in the overall process and $5 \mathrm{~m}$ behind excavation face. ("Overall process" and "5 $\mathrm{m}$ behind excavation face" in legend mean the displacements generated during overall excavation process and $5 \mathrm{~m}$ after the excavation face passed the monitoring sections, respectively) 


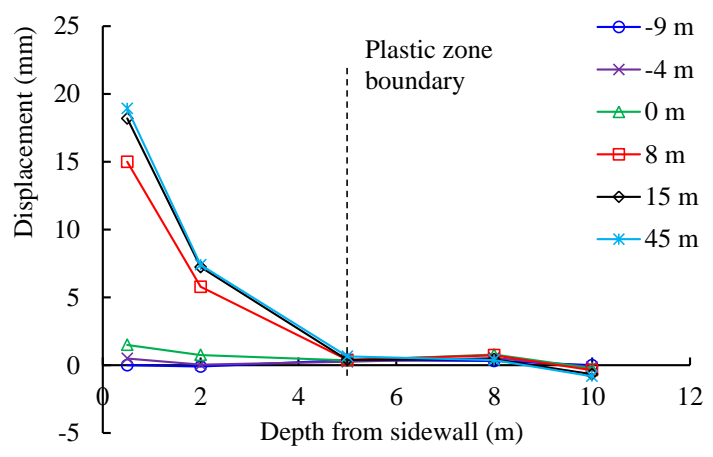

(a) Monitoring section 1

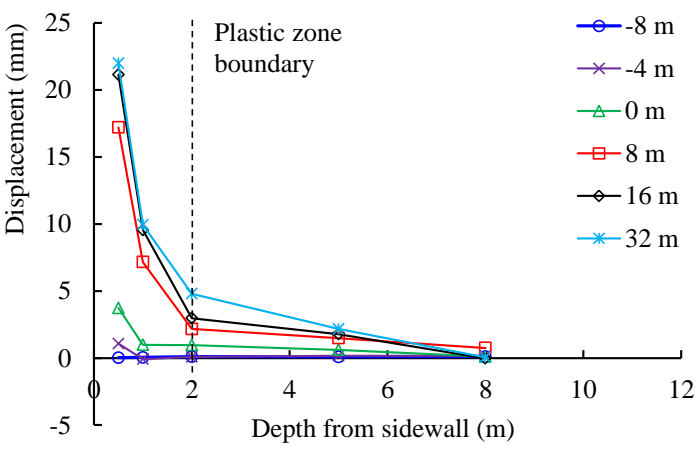

(b) Monitoring section 2

Fig. 8 First assessment of the boundary of plastic zone. (The monitoring results form the multi-point extensometers were replotted to compare the displacement gradients between adjacent measuring points for a first assessment of the boundary of plastic zone size. e.g. "- $8 \mathrm{~m}$ " in legend means the excavation face is behind the monitoring section. However, the results are just first assessment due to its discrete nature and needs further validation) 


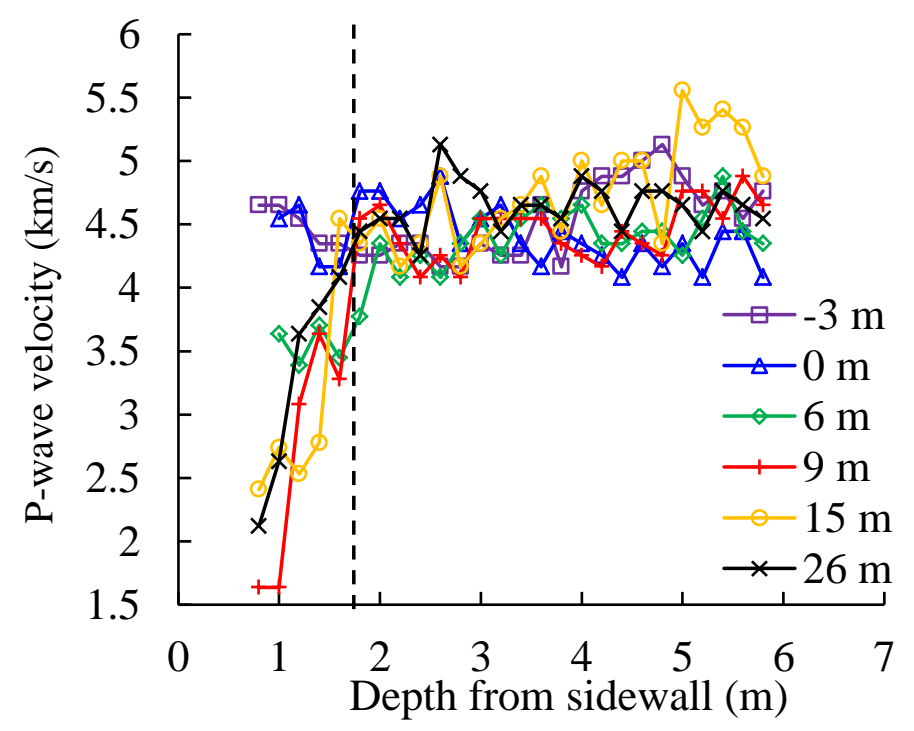

(a) Monitoring section 1

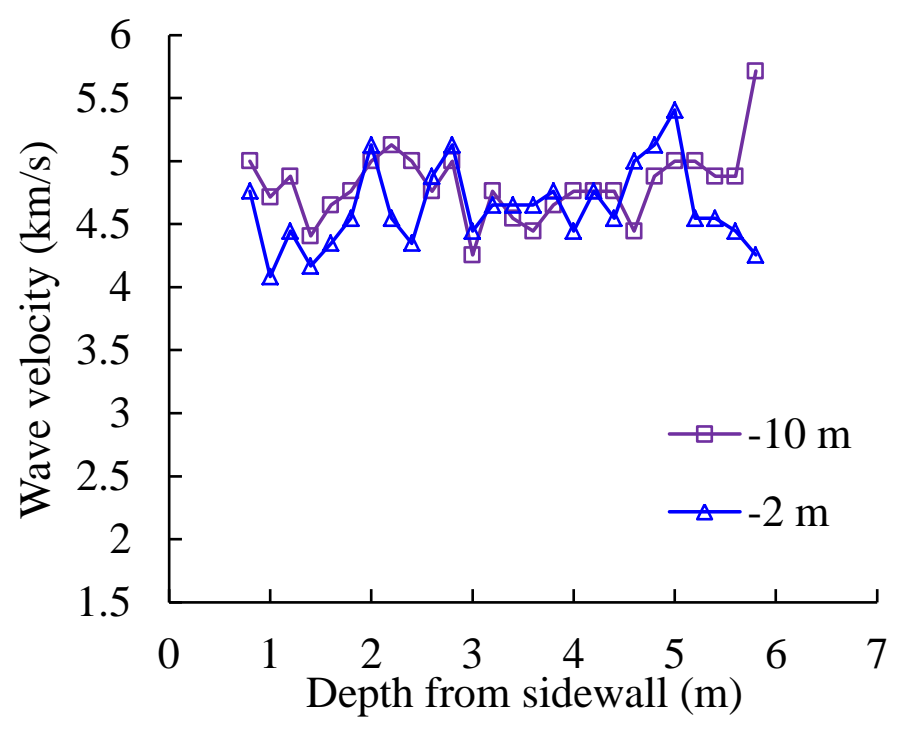

(b) Monitoring section 2

Fig. 9 P-wave velocity evolutions at two monitoring sections. (At monitoring section 1 (Fig. 9(a)), three evolution phrases of wave velocity were observed in the surrounding rock mass near sidewall during the excavation process: unchanged phrase, sharp falling phrase, stabilization phrase. Unfortunately, at monitoring section 2 (Fig. 9(b)), there was no significant variations in wave velocity observed due to the lack of data after the excavation face reached at the monitoring section). 